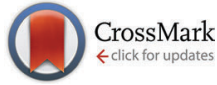

Cite this: Chem. Commun., 2014, 50, 14613

Received 22nd August 2014 Accepted 6th October 2014

DOI: $10.1039 / c 4 c c 06612 j$

www.rsc.org/chemcomm

\section{Long wavelength optical control of glutamate receptor ion channels using a tetra-ortho-substituted azobenzene derivative $\uparrow$}

\author{
A. Rullo, $\ddagger^{a}$ A. Reiner $\ddagger^{b}$ A. Reiter, ${ }^{c}$ D. Trauner, ${ }^{c}$ E. Y. Isacoff ${ }^{b}$ and G. A. Woolley $\star^{a}$
}

\begin{abstract}
A tetra-ortho-chloro substituted azobenzene unit was incorporated into a photoswitchable tethered ligand for ionotropic glutamate receptors. This compound confers the modified protein with the unusual optical responses of the substituted azo scaffold permitting channel opening with yellow and red light and channel closing with blue light.
\end{abstract}

Remote control of protein function using light affords a general method for controlling cellular chemistry. ${ }^{1,2}$ Glutamate receptors represent one of the most exciting targets for such studies due to their central roles in neurophysiology together with the difficulty of using traditional pharmacological or genetic methods to investigate their functions in an adequately selective manner., Photoswitchable tethered ligands (PTLs) based on azobenzene have emerged as a powerful optochemical-genetic tool for probing function of both ionotropic and metabotropic glutamate receptors in a range of cell types. ${ }^{5,6}$ In addition to the high specificity for their engineered receptor targets, they provide remarkable temporal precision. ${ }^{7}$ However, for manipulating the function of these key targets in vivo, the photochemical properties of current tethered ligands are less than ideal. ${ }^{8}$ Specifically, photoswitches that absorb at longer wavelengths while simultaneously exhibiting thermal bi-stability would allow greater tissue penetration by light while maximizing the degree of optical control. ${ }^{9,10}$

Recently, we discovered that certain azobenzenes substituted at all four positions ortho to the azo group exhibit an unusual combination of long wavelength photoswitching and

\footnotetext{
${ }^{a}$ Department of Chemistry, University of Toronto, Toronto M5S 3H6, Canada. E-mail: awoolley@chem.utoronto.ca

${ }^{b}$ Department of Molecular and Cell Biology, University of California, Berkeley, California, USA

cDepartment of Chemistry and Center for Integrated Protein Science, University of Munich, Munich, Germany

$\dagger$ Electronic supplementary information (ESI) available: Details of synthetic procedures. Detailed electrophysiological characterization of the toCl-MAG1 modified LiGluR channel. See DOI: 10.1039/c4cc06612j

\# These authors contributed equally.
}

thermal stability. ${ }^{9,11-14}$ Since structure-activity relationships for PTLs targeting glutamate receptors have not been extensively explored, it was unclear whether this substitution pattern would be tolerated. ${ }^{6,8,15}$ Molecular dynamics simulations suggest that the relative occupancy of the binding pocket by the two photoisomers and the degree of clamshell closure that is possible given the disposition of the linker are key factors for PTL function, both of which could be influenced by tetra-ortho functionalization of the azo group. ${ }^{16,17}$ Second, although tetraortho functionalized azobenzenes behave well in model systems, their photochemistry can be significantly influenced by the steric requirements and local polarity of their environment. ${ }^{9}$ Thus a key issue is whether tetra-ortho substitution of the azo group produces a functional glutamate receptor PTL. If it does, the possibilities for expanding the palette of PTLs with customized optical properties would be significantly enhanced.

To test function in living cells, we first had to revisit the synthetic route to the target PTL since the tetra-ortho substitution pattern alters the chemical reactivity of the azobenzene core. While tetra-ortho-methoxy substituted azobenzenes exhibit the longest wavelength switching reported to date, these compounds are particularly acid sensitive as well as being sensitive to mild reductants. ${ }^{9}$ We opted therefore to first target the tetra-orthochloro substituted species toCl-MAG1 (Scheme 1). While still somewhat acid sensitive, the tetra-ortho-chloro species shows greater stability to reduction and has enabled red-light switching in test compounds in vivo. ${ }^{9}$

Since the target compound is an asymmetrically substituted azobenzene derivative, we first explored selectively functionalizing the asymmetric tetra-ortho-chloro azobenzene derivative (1) synthesized previously. ${ }^{9}$ While the amino functionality of 1 could be elaborated by connecting a glycine spacer, the nitro group appeared to activate the system to nucleophilic aromatic substitution as well as to attack at the azo group by any unhindered nucleophile. Careful reduction of the nitro group with sodium sulphide produced 2 , which proved to be much less susceptible to attack. Compound 2 could also be produced by a diazo coupling reaction. Amide coupling of 2 with Fmoc-Gly, separation of the mono-substituted 


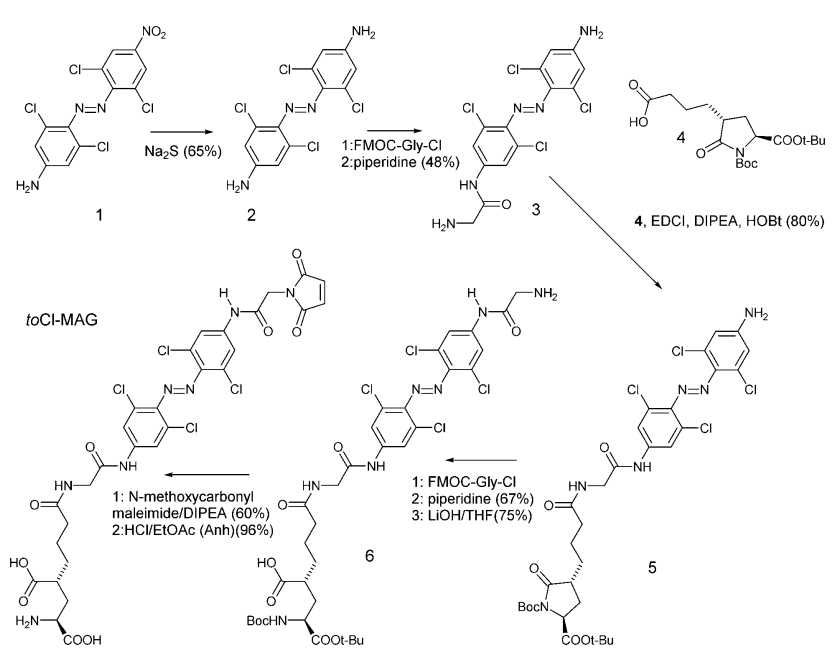

Scheme 1

product and removal of Fmoc with piperidine produced 3. The alkylamino group of $\mathbf{3}$ was then coupled with the protected glutamate derivative $\mathbf{4}$ (prepared as described previously, except that the carboxylic acid functional group of the pyroglutamic acid fragment was protected as a $t$-butyl ester). ${ }^{5,18}$ Coupling of a second Fmocprotected Gly unit on the other side of the azobenzene core, followed by deprotection and ring opening with $\mathrm{LiOH}$ produced 6 . The maleimide unit was then installed using $N$-methoxycarbonylmaleimide. Careful deprotection of the Boc groups using $\mathrm{HCl}$ in anhydrous ethyl acetate led to the final product toCl-MAG1.

Fig. 1 shows UV-Vis spectra recorded for toCl-MAG1 in phosphate buffer $\mathrm{pH} 7.0$ together with the parent MAG1 spectra for comparison. As expected, the dark-adapted trans form of the compound exhibited a peak near $470 \mathrm{~nm}$ ( $\mathrm{n}-\pi^{*}$ transition) with a tail that extends to wavelengths $>600 \mathrm{~nm}$. Irradiation with red light $(630 \mathrm{~nm})$ produced the cis isomer $\left(\mathrm{n}-\pi^{*}, 455 \mathrm{~nm}\right)$. Irradiation with blue light $\left(\lambda_{\max } 450 \mathrm{~nm}\right)$ then restored the trans

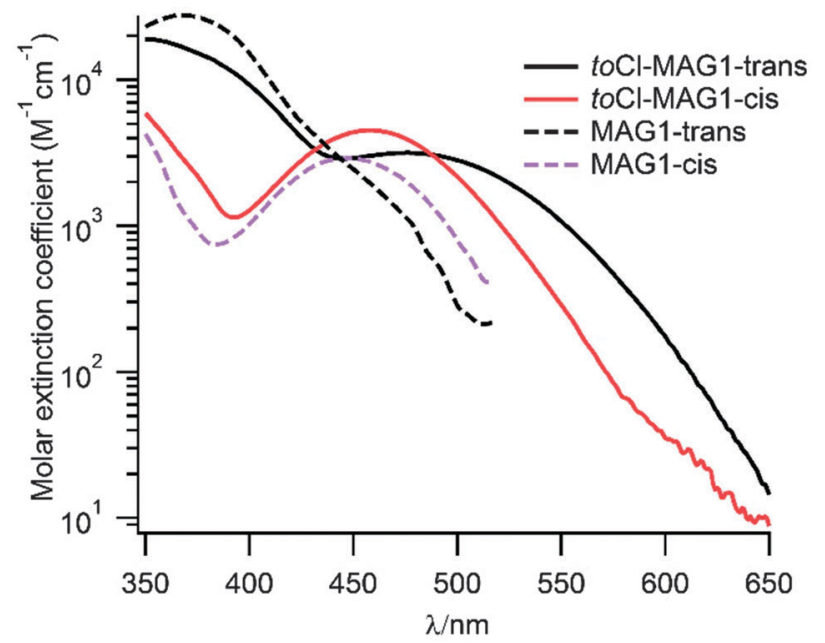

Fig. 1 UV-Vis spectra of dark-adapted toCl-MAG1 in phosphate buffer $\mathrm{pH} 7.0$ (black line), and after irradiation with red light $(630 \mathrm{~nm})$ until the photo stationary state is reached (red line). The parent PTL chromophore (without ortho substituents) is shown for comparison. state (not shown). Thermal relaxation from cis to trans occurred with a half-life of $3.5 \mathrm{~h}$ at $37{ }^{\circ} \mathrm{C}^{9}$ so that both isomers may be considered thermally stable on the time frame of typical neurophysiological experiments.

Next, we tested the function of toCl-MAG1 in living HEK293 cells expressing LiGluR, a modified kainate receptor GluK2(439C)

(a)

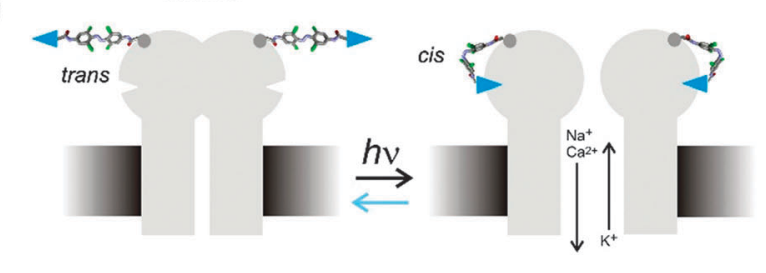

(b)

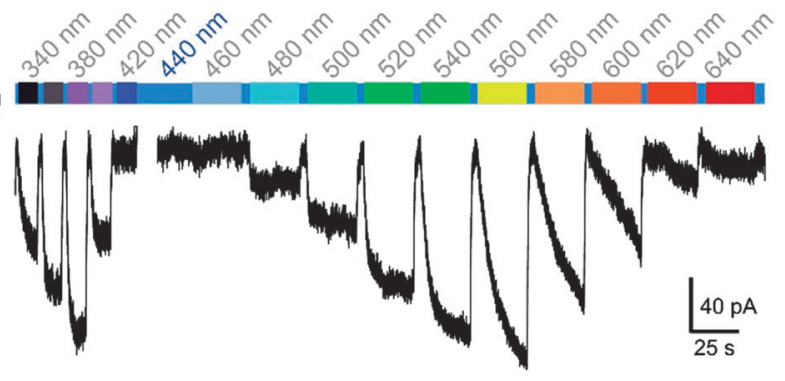

(c)
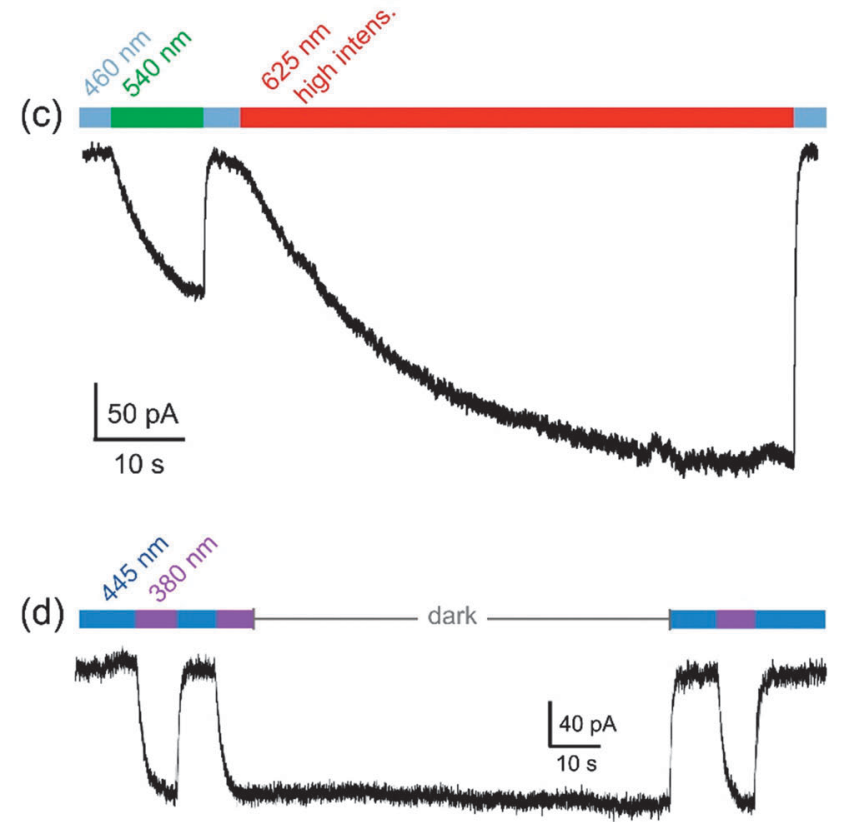

Fig. 2 (a) Schematic diagram of optical control of channel opening/closing using toCl-MAG1. (b) Photo-activation of LiGluR at different wavelengths ranging from $340 \mathrm{~nm}$ to $640 \mathrm{~nm}$. Opening in response to light (colour indicated in the bar above) is reflected in a downward deflection in the current trace. Brief blue light $(440 \mathrm{~nm}$ ) pulses (indicated as blue vertical lines) close the channel. At wavelengths $>540 \mathrm{~nm}$ the photo-stationary state (PSS) is not reached within $30 \mathrm{~s}$ with the light intensity used $\left(\sim 1 \mathrm{~mW} \mathrm{~mm}^{-2}\right)$. For details

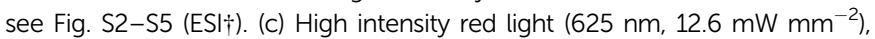
produces a PSS in less than $1 \mathrm{~min}$. Here, the photocurrent amplitude is considerably higher than at the $540 \mathrm{~nm}$ PSS ((2.08 \pm 0.12$)$-fold photocurrent, mean \pm s.d., $n=5$ cells). (d) In the dark, activated channels remain open for extended periods of time (interrupted bar, $>80 \mathrm{~s}$ ), indicating that thermal cis-to-trans relaxation happens on much slower timescales. 
reported previously. ${ }^{5}$ Channel opening is measured as a downward deflection in the whole-cell current (Fig. 2). After toCl-MAG1 labelling of LiGluR we observed reversible and efficient photoswitching, similar to unsubstituted MAG1. ${ }^{5}$ toCl-MAG1 triggered channel opening with UV light, channel closing in the blue wavelength range, and for the first time, activation in the yellow and even the red region of the spectrum (Fig. 2; Fig. S1-S4, ESI $\dagger$ ). As expected, the photoswitch was bi-stable, i.e. no relaxation from cis-to-trans was observed in the dark on the timescale of minutes (Fig. 2d).

These data show that the tetra-ortho substitution of the azobenzene scaffold is well tolerated and does not negatively impact optical control of channel opening by adversely affecting ligand accessibility or conformational dynamics. Photoswitching was fully reversible over an extended period of time, indicating excellent photostability of the toCl-MAG1 ligand (Fig. S6, ESI $\dagger$ ). The optical response conferred on the glutamate receptor channel by toCl-MAG1 is essentially in accordance with the absorption behaviour of the isolated ligand as well as for the tetra-orthochloro azobenzene chromophore attached via $p$-amido groups to peptides. ${ }^{9}$ In contrast to channel rhodopsin-based light gated channels where the chromophore is embedded in the core of the protein, in this system significant chemical changes in the chromophore can apparently be made while preserving the gating response of the channel. This property of the system implies that a range of chromophores with optical responses tuned for specific applications may be developed and applied in a modular fashion.

Fast photoswitching of toCl-MAG1 was observed for deactivation at $\sim 460 \mathrm{~nm}$ while activation in the UV and green regions of the spectrum (Fig. S2 and S5, ESI $\dagger$ ) was $\sim 2$-fold slower than switching of unsubstituted MAG1 at comparable light intensities $\left(0.2-1 \mathrm{~s}^{-1}\right.$, at $\left.1 \mathrm{~mW} \mathrm{~mm}^{-2}\right)$. Switching with red light was slower, as expected due to the low absorbance of the PTL in this region (Fig. 1 and 2), however, the photostationary state produced with high intensity red light $\left(625 \mathrm{~nm}, 12.6 \mathrm{~mW} \mathrm{~mm}^{-2}\right.$ ) leads to large photocurrents (Fig. 2c). Since the switch is thermally stable in the cis isomeric form, red light may be used to confer a basal shift in the resting potential of cells. Alternatively, to achieve fast switching, red light could be used at even higher intensities. Our results clearly establish the tetra-ortho substitution pattern as a viable platform for further development of the photo-pharmacology of glutamate receptors. For example, tetra-ortho substituted azobenzenes with $p$-amino groups have recently been found to exhibit efficient red light switching via their azonium forms. ${ }^{12}$ The data reported here suggest such azonium ions too could be incorporated into PTLs for LiGluR, further extending the options for optical manipulation of these channels in vivo.

A tetra-ortho-chloro substituted azobenzene scaffold is well tolerated as a replacement for azobenzene itself in a photoswitchable tethered ligand for the ionotropic glutamate receptor. The substitution permits optical control of ion channel opening with yellow and red light for the first time. The ability of the system to tolerate such modifications indicates that a variety of optical responses can be produced via rational chemical design and modification of the azobenzene core.

\section{Notes and references}

1 S. Szobota and E. Y. Isacoff, Annu. Rev. Biophys. Biomol. Struct., 2010, 39, 329-348.

2 P. Hegemann and A. Moglich, Nat. Methods, 2011, 8, 39-42.

3 C. M. Niswender and P. J. Conn, Annu. Rev. Pharmacol. Toxicol., 2010, 50, 295-322.

4 S. F. Traynelis, L. P. Wollmuth, C. J. McBain, F. S. Menniti, K. M. Vance, K. K. Ogden, K. B. Hansen, H. Yuan, S. J. Myers and R. Dingledine, Pharmacol. Rev., 2010, 62, 405-496.

5 M. Volgraf, P. Gorostiza, R. Numano, R. H. Kramer, E. Y. Isacoff and D. Trauner, Nat. Chem. Biol., 2006, 2, 47-52.

6 J. Levitz, C. Pantoja, B. Gaub, H. Janovjak, A. Reiner, A. Hoagland, D. Schoppik, B. Kane, P. Stawski, A. F. Schier, D. Trauner and E. Y. Isacoff, Nat. Neurosci., 2013, 16, 507-516.

7 A. Reiner and E. Y. Isacoff, Nat. Chem. Biol., 2014, 10, 273-280.

8 M. A. Kienzler, A. Reiner, E. Trautman, S. Yoo, D. Trauner and E. Y. Isacoff, J. Am. Chem. Soc., 2013, 135, 17683-17686.

9 S. Samanta, A. A. Beharry, O. Sadovski, T. M. McCormick, A. Babalhavaeji, V. Tropepe and G. A. Woolley, J. Am. Chem. Soc., 2013, 135, 9777-9784.

10 G. A. Woolley and A. A. Beharry, Chem. Soc. Rev., 2011, 40, $4422-4437$.

11 A. A. Beharry, O. Sadovski and G. A. Woolley, J. Am. Chem. Soc., 2011, 133, 19684-19687.

12 S. Samanta, A. Babalhavaeji, M. X. Dong and G. A. Woolley, Angew. Chem., Int. Ed., 2013, 52, 14127-14130.

13 S. Samanta, T. M. McCormick, S. K. Schmidt, D. S. Seferos and G. A. Woolley, Chem. Commun., 2013, 49, 10314-10316.

14 D. Bleger, J. Schwarz, A. M. Brouwer and S. Hecht, J. Am. Chem. Soc., 2012, 134, 20597-20600.

15 M. Izquierdo-Serra, M. Gascon-Moya, J. J. Hirtz, S. Pittolo, K. E. Poskanzer, E. Ferrer, R. Alibes, F. Busque, R. Yuste, J. Hernando and P. Gorostiza, J. Am. Chem. Soc., 2014, 136, 8693-8701.

16 T. Wolter, T. Steinbrecher, D. Trauner and M. Elstner, PLoS One, 2014, 9, e92716.

17 R. Numano, S. Szobota, A. Y. Lau, P. Gorostiza, M. Volgraf, B. Roux, D. Trauner and E. Y. Isacoff, Proc. Natl. Acad. Sci. U. S. A., 2009, 106, 6814-6819.

18 J. Ezquerra, C. Pedregal, A. Rubio, B. Yruretagoyena, A. Escribano and F. Sanchezferrando, Tetrahedron, 1993, 49, 8665-8678. 